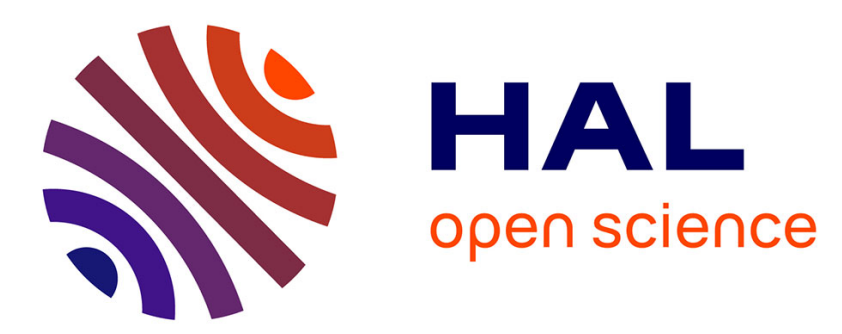

\title{
Approximating optimal control problems governed by variational inequalities
}

\author{
Viorel Barbu, P. Neittaanmaki, A. Niemisto
}

\section{To cite this version:}

Viorel Barbu, P. Neittaanmaki, A. Niemisto. Approximating optimal control problems governed by variational inequalities. [Research Report] RR-1636, INRIA. 1992. inria-00074925

\section{HAL Id: inria-00074925 \\ https://hal.inria.fr/inria-00074925}

Submitted on 24 May 2006

HAL is a multi-disciplinary open access archive for the deposit and dissemination of scientific research documents, whether they are published or not. The documents may come from teaching and research institutions in France or abroad, or from public or private research centers.
L'archive ouverte pluridisciplinaire HAL, est destinée au dépôt et à la diffusion de documents scientifiques de niveau recherche, publiés ou non, émanant des établissements d'enseignement et de recherche français ou étrangers, des laboratoires publics ou privés. 


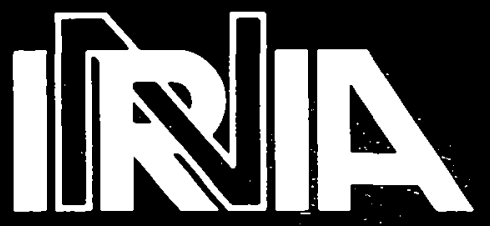

UNITÉ DE RECHERCHE INRIA-ROCOUENCOURT

\section{Rapports de Recherche}

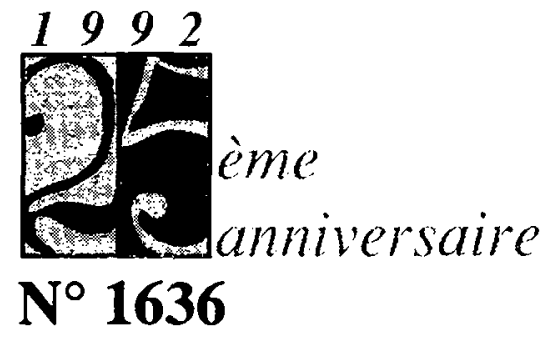

Programme 5

Traitement du Signal, Automatique et Productique

\section{APPROXIMATING OPTIMAL CONTROL PROBLEMS GOVERNED BY VARIATIONAL INEQUALITIES}

Institut National de Recherche en Informatique et en Automatique

Domaine de Voluceau

Rocquencourt

$$
\text { B.P.105 }
$$

78153 LeChesnay Cedex

France

Tél.:(1) 39635511

\section{Viorel BARBU \\ Pekka NEITTAANMÄKI \\ Antti NIEMISTÖ}


Approximation de problèmes de contrôle optimal gouvernés par des inéquations variationnelles

\title{
Approximating optimal control problems governed by variational inequalities
}

\author{
Viorel Barbu ${ }^{1}$, Pekka Neittaanmäk ${ }^{2}$ and Antti Niemistö ${ }^{2}$ \\ 1 University of Iaşi, 6600 Iaşi, Romania \\ 2 University of Jyväskylä, P.O. Box 35 \\ 40351 Jyväskylä, Finland
}

\section{RÉSUMÉ}

On introduit un algorithme pour la resolution de problèmes de contrôle optimal gouvernés par des inéquations variationelles elliptiques. On donne quelques applications et examples numeriques.

\section{ABSTRACT}

It is proposed an approximating method for optimal control problems governed by elliptic variational inequalities. Some applications and numerical examples are treated.

MOTS CLÉS

Contrôl optimal, inéquations variationelles, algorithme pour la resolution.

\section{KEY WORDS}

Optimal control, variational inequalities, approximating method. 


\section{INTRODUCTION}

Consider the optimal control problem

$$
\min _{u \in U}\{g(y)+h(u) ; A y+\partial \varphi(y) \ni B u+f\},
$$

where

(i) $A$ is a single valued maximal monotone operator in a real Hilbert space $H$ and $\partial \varphi$ : $H \rightarrow H$ is the subdifferential of a lower semicontinuous convex function $\varphi: H \rightarrow \overline{\mathbf{R}}=$ ] $-\infty,+\infty$ ]. Moreover, there exists $C \in \mathbf{R}$ such that

$$
\varphi\left((I+\lambda A)^{-1} y\right) \leq \varphi(y)+C \lambda \quad \forall y \in H, \forall \lambda>0 .
$$

Here $A_{\lambda}=\lambda^{-1}\left(I-(I+\lambda A)^{-1}\right)$ is the Yosida approximation of $A$ (see e.g. [2], [4]).

(ii) $A^{-1}$ is compact, i.e., for any sequence $\left[y_{n}, z_{n}\right] \in A$ such that $\left\{z_{n}\right\}$ is bounded in $H$, $\left\{y_{n}\right\}$ is compact in $H$.

(iii) $g: H \rightarrow \overline{\mathbf{R}}$ and $h: U \rightarrow \overline{\mathbf{R}}$ are convex, lower semicontinuous, $g(y) \geq C \quad \forall y \in H$ and

$$
h(u) \geq \gamma|u|_{U}+C \quad \forall u \in U
$$

for some $\gamma>0$.

(iv) $B$ is a linear continuous operator from a real Hilbert space $U$ to $H$ and $f \in H$.

We have denoted by $|\cdot|$ and $|\cdot| U$ the norm of $H$ and $U$, respectively. The scalar products in $H$ and $U$ will be denoted by $(\cdot, \cdot)$ and $\langle\cdot, \cdot\rangle$, respectively.

Following an idea developed in [3] we shall approximate here problem (1.1), which under our assumptions has at least one solution $\left(y^{*}, u^{*}\right)$ by the following one

$$
\begin{gathered}
\min \left\{g(y)+h(u)+\frac{1}{\varepsilon}\left(\varphi(y)+\varphi^{*}(v)-(y, v)\right) ;\right. \\
A y=B u-v+f ; u \in U, v \in H\},
\end{gathered}
$$

were $\varphi^{*}: H \rightarrow H$ is the conjugate of $\varphi$, i.e

$$
\varphi^{*}(v)=\sup \{(y, v)-\varphi(y) ; y \in H\}, \quad \forall v \in H .
$$

The convergence of this approximating process and the numerical implementation of the resulting algorithm in the case of an optimal control problem governed by the obstacle problem associated with the loaded beam represent much of the substance of this paper. 


\section{EXISTENCE AND CONVERGENCE OF TIIE APPROXIMATING PROBLEM}

We shall prove here the following result.

THEOREM 1. For every $\varepsilon>0$ problem (1.4) has at least one solution $\left(y_{\varepsilon}, v_{\varepsilon}, u_{\varepsilon}\right) \in H \times H \times$ $U$. The set $\left\{\left(y_{\varepsilon}, v_{\varepsilon}, u_{\varepsilon}\right)\right\}_{\varepsilon}>0$ is compact in $H \times H_{w} \times U_{w}$ and every limit point $\left(y^{*}, v^{*}, u^{*}\right)$ for $\varepsilon \rightarrow 0$ is a solution to problem (1.1). Moreover,

$$
\lim _{\varepsilon \rightarrow 0}\left(g\left(y_{\varepsilon}\right)+h\left(u_{\varepsilon}\right)\right)=\inf \{g(y)+h(u) ; A y+\partial \varphi(y) \ni B u+f ; u \in U\}
$$

Here $H_{w}$ and $U_{w}$ are the spaces $H$ and $U$ endowed with the weak topology.

ProOF: Let

$$
\begin{gathered}
d=\inf \left\{g(y)+h(u)+\frac{1}{\varepsilon}\left(\varphi(y)+\varphi^{*}(v)-(y, v)\right) ;\right. \\
A y=B u-v+f, \quad u \in U, v \in H\} .
\end{gathered}
$$

(We note that by assumption (ii) the equation $A y \ni w$ has for every $w \in H$ at least one solution.) It is readily seen that $d>-\infty$. Now let $\left(y_{n}, u_{n}, v_{n}\right)$ be such that

$$
d \leq g\left(y_{n}\right)+h\left(u_{n}\right)+\frac{1}{\varepsilon}\left(\varphi\left(y_{n}\right)+\varphi^{*}\left(v_{n}\right)-\left(y_{n}, v_{n}\right)\right) \leq d+\frac{1}{n}
$$

By assumption (iii), $\left\{u_{n}\right\}$ is bounded in $U$ and so on a subsequence, again denoted $n$,

$$
u_{n} \longrightarrow u_{\varepsilon} \text { weakly in } U \text {. }
$$

On the other hand, by (1.5) we have

$$
\varphi^{*}\left(v_{n}\right) \geq-\varphi(y)+\left(v_{n}, y\right) \quad \forall y \in H
$$

and therefore

$$
g\left(y_{n}\right)+h\left(u_{n}\right) \leq d+\frac{1}{n} ; \quad \varphi\left(y_{n}\right)+\left(v_{n}, y-y_{n}\right)-\varphi(y) \leq C .
$$

For $y=(I+\varepsilon A)^{-1} y_{n}$ we get in virtue of hypothesis (1.2)

$$
-\left(v_{n}, A_{\varepsilon} y_{n}\right) \leq C
$$

and therefore

$$
\left(A y_{n}, A_{\varepsilon} y_{n}\right) \leq C\left|A_{\varepsilon} y_{n}\right| \quad \forall \varepsilon>0
$$

and by the monotonicity of $A$ this implies that

$$
\left|A_{\varepsilon} y_{n}\right| \leq C \quad \forall n, \varepsilon>0
$$


Finally, it follows that

$$
\left|A y_{n}\right| \leq C \quad \forall n .
$$

Here $\left\{v_{n}\right\}$ is bounded in $H$ and so on a subsequence, we have

$$
\begin{array}{cl}
v_{n} \longrightarrow v_{\varepsilon} & \text { weakly in } H \\
y_{n} \longrightarrow y_{\varepsilon} & \text { strongly in } H \\
A y_{n} \longrightarrow A y_{e} & \text { weakly in } H
\end{array}
$$

Then letting $n$ tend to $+\infty$ in (2.1) we get that $\left(y_{\varepsilon}, u_{\varepsilon}, v_{\varepsilon}\right)$ is a solution to (1.4).

Next we have

$$
g\left(y_{\varepsilon}\right)+h\left(u_{\varepsilon}\right) \leq g\left(y^{*}\right)+h\left(u^{*}\right) \quad \forall \varepsilon>0
$$

and

$$
\varphi\left(y_{\varepsilon}\right)+\varphi^{*}\left(v_{\varepsilon}\right)-\left(y_{\varepsilon}, v_{\varepsilon}\right) \leq C \varepsilon \quad \forall \varepsilon>0,
$$

where $\left(y^{*}, u^{*}\right)$ is a solution to problem (1.1).

We have therefore

$$
\varphi\left(y_{\varepsilon}\right)-\varphi(y)+\left(y-y_{\varepsilon}, v_{\varepsilon}\right) \leq C \varepsilon \quad \forall y \in H
$$

and for $y=\left(I+n^{-1} A\right)^{-1} y_{\varepsilon}$ we get letting $n \rightarrow+\infty$

$$
-\left(A y_{\varepsilon}, v_{\varepsilon}\right) \leq C \quad \forall \varepsilon>0 .
$$

Then multiplying the equation $A y_{\varepsilon}=B u_{\varepsilon}-v_{\varepsilon}+f$ by $A y_{\varepsilon}$ we get

$$
\left|A y_{\varepsilon}\right| \leq C \quad \forall \varepsilon>0 .
$$

Hence $\left\{y_{\varepsilon}\right\},\left\{u_{\varepsilon}\right\}$ and $\left\{v_{\varepsilon}\right\}$ are bounded. Moreover by assumption (ii) we know that $\left\{y_{\varepsilon}\right\}$ is compact in $H$. We have therefore shown that $\left\{\left(y_{\varepsilon}, v_{\varepsilon}, u_{\varepsilon}\right)\right\}$ is compact in $H \times H_{w} \times U_{w}$. Let $\left\{\varepsilon_{n}\right\} \rightarrow 0$ be such that

$$
\begin{array}{ll}
y_{\varepsilon_{n}} \longrightarrow \bar{y} & \text { strongly in } H, \\
u_{\varepsilon_{n}} \longrightarrow \bar{u} & \text { weakly in } U, \\
v_{\varepsilon_{n}} \longrightarrow \bar{v} & \text { weakly in } H .
\end{array}
$$

Then by (2.3) we see that

$$
\varphi(\bar{y})+\varphi^{*}(\bar{v})-(\bar{y}, \bar{v})=0
$$

i.e., $\bar{v} \in \partial \varphi(\bar{y})$. Since $g(\bar{y})+h(\bar{u}) \leq g\left(y^{*}\right)+h\left(u^{*}\right)=\inf (1.1)$ we conclude that $(\bar{y}, \bar{u})$ is optimal in problem (1.1). It is also clear that $g\left(y_{\varepsilon}\right)+h\left(u_{\varepsilon}\right) \rightarrow \inf (1.1)$ thereby completing the proof. 


\section{First order NECESSARy CONDITIONS OF optimality For PROBLEM (1.4)}

We shall assume here that $W$ is a reflexive Banach space such that $W \subset H \subset W^{\prime}$ algebraically and topologically where $W^{\prime}$ is the dual of $W$ and the injection of $W$ to $H$ is compact.

Let $A$ be a continuously differentiable monotone operator from $W$ to $W^{\prime}$ and denote again $A$ the operator $A_{H}: D(A) \subset H \rightarrow H$ defined by

$$
A_{H} y=A y \quad \forall y \in D(A)=\{y \in W ; A y\}
$$

We will also assume that

$$
\begin{aligned}
& (A y, y) \geq w\|y\|^{p}+C \quad \forall y \in W, \quad p \geq 2 \\
& A^{-1} \text { is compact from } H \text { to } W \\
& \left(A_{y}(y) p, p\right) \geq w_{0}\|p\|^{2} \quad \forall p \in W
\end{aligned}
$$

where $A_{y}$ is the Fréchet (Gâteaux) derivative of $A \in C^{1}\left(W, W^{\prime}\right)$ and $\|\cdot\|$ is the norm of $W$.

It is clear that if (1.2) holds then $A=A_{H}$ satisfies assumptions (i) and (ii). We shall also assume that assumptions (iii) and (iv) are satisfied and $g$ is finite on $H$.

TheOREM 2. Let $\left(y^{*}, u^{*}, v^{*}\right) \in W \times U \times H$ be optimal in problem (1.4). Then there exists $p \in W$ such that

$$
\begin{aligned}
& -\left(A_{y}\left(y^{*}\right)\right)^{*} p \in \partial g\left(y^{*}\right)+\varepsilon^{-1}\left(\partial^{*} \varphi\left(y^{*}\right)-v^{*}\right), \\
& 0 \in \varepsilon p+\partial \varphi^{*}\left(v^{*}\right)-y^{*}, \\
& B^{*} p \in \partial h\left(u^{*}\right) .
\end{aligned}
$$

Here $\left(A_{y}\right)^{*}$ is the adjoint of $A_{y}$ and $\partial g: H \rightarrow H, \partial \varphi^{*}: H \rightarrow H, \partial h: U \rightarrow U$ are the subdifferentials of $g, \varphi^{*}$ and $h$, respectively.

$\partial^{*} \varphi: W \rightarrow W^{\prime}$ is the subdifferential of $\varphi$ viewed as a function from $W$ to $\overline{\mathbf{R}}$, i.e.

$$
\partial^{*} \varphi(y)=\left\{w \in W^{\prime} ; \varphi(y) \leq \varphi(z)+(w, y-z) \quad \forall z \in W\right\}
$$

We note that $\partial^{*} \varphi$ is an extension of $\partial \varphi$.

Proof: We follow the standard arguments (see e.g. [1], [2]).

Consider the approximating problem

$$
\begin{gathered}
\min \left\{g_{\lambda}(y)+h(u)+\frac{1}{\varepsilon}\left(\varphi_{\lambda}(y)+\left(\varphi_{\lambda}\right)^{*}(v)-(y, v)\right)+\frac{1}{2}\left(\left|u-u^{*}\right|_{U}^{2}+\left|v-v^{*}\right|^{2}\right)\right. \\
A y=B u-v+f, u \in U, v \in H, \quad y \in W\}
\end{gathered}
$$

where $g_{\lambda}$ and $\varphi_{\lambda}$ are the regularizations of $g$ and $\varphi$ (see [2, pp. 121]. 
Problem (3.8) has at least one solution and for $\lambda \rightarrow 0$ we have (see e.g. [1], [2])

$$
\begin{array}{cl}
u_{\lambda} \longrightarrow u^{*} & \text { strongly in } U, \\
v_{\lambda} \longrightarrow v^{*} & \text { strongly in } H, \\
A y_{\lambda} \longrightarrow A y^{*} & \text { strongly in } H, \\
y_{\lambda} \longrightarrow y^{*} & \text { strongly in } H, \text { and weakly in } W .
\end{array}
$$

On the other hand, since $A, g_{\lambda}$ and $\varphi_{\lambda}$ are differentiable we get for problem (3.8) the optimality system

$$
\begin{aligned}
& -A_{y}^{*}\left(y_{\lambda}\right) p_{\lambda}=\nabla g_{\lambda}\left(y_{\lambda}\right)+\frac{1}{\varepsilon}\left(\partial \varphi_{\lambda}\left(y_{\lambda}\right)-v_{\lambda}\right), \\
& y_{\lambda}-\varepsilon p_{\lambda} \in \partial \varphi_{\lambda}^{*}\left(v_{\lambda}\right) \Longleftrightarrow v_{\lambda} \in \partial \varphi_{\lambda}\left(y_{\lambda}-\varepsilon p_{\lambda}\right), \\
& \beta^{*} p_{\lambda} \in \partial h\left(u_{\lambda}\right)
\end{aligned}
$$

Multiplying equation (3.10) by $p_{\lambda}$ and using condition (3.2) we get that $\left\{p_{\lambda}\right\}$ is bounded in $W$ because $\nabla g_{\lambda}$ is uniformly bounded on bounded subsets and $\partial \varphi_{\lambda}$ is monotone. Hence on a subsequence, again denoted $\lambda$ we have

$$
\begin{array}{cl}
p_{\lambda} \longrightarrow p & \text { weakly in } W, \text { strongly in } H, \\
A_{y}^{*}\left(y_{\lambda}\right) p_{\lambda} \longrightarrow A_{y}^{*}\left(y^{*}\right) p & \text { weakly in } W^{\prime}, \\
\nabla g_{\lambda}\left(y_{\lambda}\right) \longrightarrow \xi \in \partial g\left(y^{*}\right) & \text { weakly in } H, \\
\nabla \varphi_{\lambda}\left(y_{\lambda}\right) \longrightarrow \eta \in \partial^{*} \varphi\left(y^{*}\right) & \text { weakly in } W^{\prime},
\end{array}
$$

and so system (3.4)-(3.6) follows letting $\lambda$ tend to zero in (3.10)-(3.12).

We note that if

$$
\varphi\left(\operatorname{Proj} \frac{y}{D(A)}\right) \leq \varphi(y) \quad \forall y \in H
$$

and

$$
\left(A^{0} y,(\partial \varphi)_{\lambda} y\right) \geq 0 \quad \forall y \in D(A), \lambda>0
$$

then assumption (1.2) holds, i.e.

$$
\varphi\left((I+\lambda A)^{-1} y\right) \leq \varphi(y) \quad \forall y \in D(A)
$$

(see e.g. [4]). Thus, the previous results are in particular applicable to optimal control problems governed by variational inequality

$$
\begin{aligned}
-\operatorname{div} a(\nabla y) & =B u+f \text { in }\{x \in \Omega ; y(x)>\psi\}, \\
-\operatorname{div} a(\nabla y) & \geq B u+f, y \geq \psi \text { a.e. in } \Omega, \\
y & =0 \text { in } \partial \Omega
\end{aligned}
$$


where $\psi \leq 0$ is a constant, $\Omega$ is an open, bounded subset of $\Omega^{*}$ with a sufficiently smooth boundary and $a: \mathbf{R}^{N} \rightarrow \mathbf{R}^{N}$ is a continuous, differentiable, monotone mapping such that

$$
\begin{aligned}
(a(\xi), \xi) & \geq w\|\xi\|_{N}^{p}+C \quad \forall \xi \in \mathbf{R}^{N}, \\
\|a(\xi)\|_{N} & \leq C_{1}\|\xi\|_{N}^{p-1}+C_{2} \quad \forall \xi \in \mathbf{R}^{N}, \\
\left(a^{\prime}(\xi) y, y\right) & \geq w_{0}\|y\|_{N}^{2} \quad \forall y, \xi \in \mathbf{R}^{N}
\end{aligned}
$$

(Here $\|\cdot\|_{N}$ is the Euclidean norm in $R^{N}$.) Then Eq. (3.14) can be written in the form

$$
A y+\partial \varphi(y) \ni B u+f
$$

where $A=-\operatorname{div} a(\nabla y), W=W_{0}^{1, p}(\Omega), H=L^{2}(\Omega)$ and

$$
\varphi(y)= \begin{cases}0 & \text { if } y(x) \geq \psi \\ +\infty & \text { otherwise. }\end{cases}
$$

It is readily seen that condition (3.13) holds in this case.

We note also that in the particular case where $\varphi=I_{K}$ (the indicator function of closed convex subset $K$ of $H$ ) problem (1.4) becomes

$$
\min _{y \in K, u \in U}\left\{g(y)+h(u)+\frac{1}{\varepsilon}\left(H_{K}(v)-(y, v)\right\},\right.
$$

where $H_{K}$ is the support function of $K$, i.e.,

$$
H_{K}(v)=\sup \left\{(y, v) ; y \in K^{r}\right\}
$$

Finally, if $W$ is a Hilbert space and $A \in L\left(W, W^{\prime}\right)$ satisfies the coercivity condition

$$
(A y, y) \geq w\|y\|^{2} \quad \forall y \in W
$$

then Theorems 1 and 2 remain valid and the optimality system (3.4) (3.6) has the following form

$$
\begin{aligned}
-A^{*} p & \in \partial g\left(y^{*}\right)+\varepsilon^{-1}\left(\partial \varphi\left(y^{*}\right)-v^{*}\right), \\
0 & \in \varepsilon p+\partial \varphi^{*}\left(v^{*}\right)-y^{*} \\
B^{*} p & \in \partial h\left(u^{*}\right) .
\end{aligned}
$$

\section{EXAMPLES AND NUMERICAL TESTS}

Next we shall present two one dimensional examples with numerical tests. In the first case our operator $A$ will be a linear differential operator of order four and in the second example it will be nonlinear of order two. In both cases the operator $B$ will be identity and the functions $h$ and $f$ will be identically zero. 


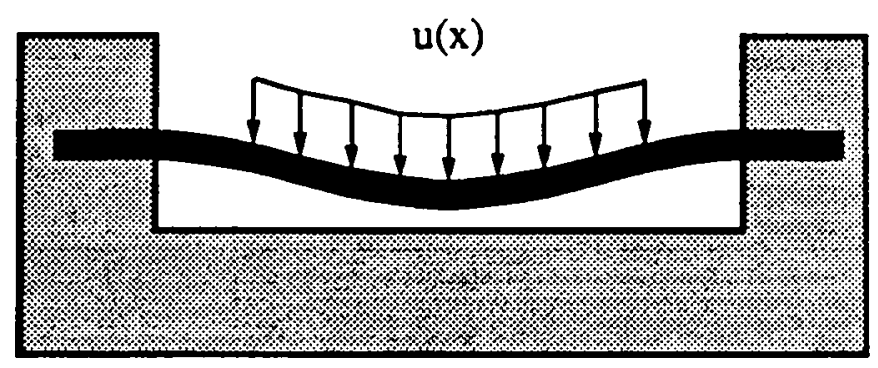

Figure 4.1.

\section{EXAMPLE 1.}

Let us consider a clamped beam the deflection of which is limited from below by a rigid obstacle described by a function $q$ (figure 4.1). The control variable expresses the physical meaning of the load of the beam. The aim is to find a load density $u$ in such a way that the contact between the beam and the obstacle will be maximized.

The problem can be formulated as follows

$$
\underset{u \in U_{a d}}{\operatorname{minimize}}\left\{g(y(u)) \equiv \frac{1}{2} \int_{0}^{1}(y(x)-q(x))^{2} d x\right\},
$$

subject to

$$
\left\{\begin{aligned}
A y+\partial \varphi(y) & \ni u \\
y & \in H_{0}^{2}((0,1))
\end{aligned}\right.
$$

where

$$
\begin{aligned}
& A y=y^{(i v)}\left(=y^{\prime \prime \prime \prime}\right), \\
& \varphi: V \rightarrow(-\infty,+\infty) \text { is given by } \\
& \varphi(y)= \begin{cases}0 & y \geq q \text { a.e. in }(0,1) \\
+\infty & \text { otherwise, }\end{cases} \\
& U_{a d}=\left\{u \in L^{\infty}((0,1)) \mid \alpha \leq u(x) \leq \beta, \text { a.e. in }(0,1), \int_{0}^{1} u(x) d x=M\right\}
\end{aligned}
$$

with given positive constants $\alpha, \beta$ and $M$.

Clearly $\left(P^{E_{1}}\right)$ satisfies the conditions (i),..,(iv) of (1.1) and we have existence for the problem. Let us formulate the corresponding problem for (1.4), which can be written as follows

$\left(P_{\varepsilon}^{E_{1}}\right)$

$$
\begin{aligned}
\underset{\substack{u \in U_{\text {ad }} \\
v \leq 0}}{\operatorname{minimize}}\left\{J_{\varepsilon}(y, v) \equiv\right. & \frac{1}{2} \int_{0}^{1}(y(x)-q(x))^{2} d x \\
& \left.\quad-\frac{1}{\varepsilon} \int_{0}^{1} v(x)(y(x)-q(x)) d x \mid y \geq q \text { a.e. }(0,1)\right\}
\end{aligned}
$$


where $y(x)$ is the weak solution for the equation

$$
\left\{\begin{aligned}
y^{i v} & =u-v \\
y & \in H_{0}^{2}((0,1)) .
\end{aligned}\right.
$$

If we apply the quadratic penalty function approach for the state constraint (i.e. we approximate the indicator function $I_{K}$ with quadratic penalty function), we get

$$
\begin{aligned}
\underset{\substack{u \in U_{a d} \\
v \leq 0}}{\operatorname{minimize}}\left\{J_{\varepsilon \delta}(y, v) \equiv\right. & \frac{1}{2} \int_{0}^{1}(y(x)-q(x))^{2} d x \\
& -\frac{1}{\varepsilon} \int_{0}^{1} v(x)(y(x)-q(x)) d x \\
& \left.+\frac{1}{2 \delta} \int_{0}^{1}\left([y(x)-q(x)]^{-}\right)^{2} d x\right\}
\end{aligned}
$$

where $y(x)$ is the weak solution of $(4.1)$ and

$$
[y(x)-q(x)]^{-}= \begin{cases}-(y(x)-q(x)) & , y(x) \leq q(x) \\ 0 & , y(x)>q(x) .\end{cases}
$$

Theorem 1 and the general optimization theory implies that the solution of $\left(P_{\varepsilon \delta}^{E_{1}}\right)$ converges to the solution of $\left(P^{E_{1}}\right)$ as $\varepsilon, \delta \rightarrow 0+$.

Let $\left(y^{*}, u^{*}, v^{*}\right)$ be optimal in $\left(P_{\varepsilon \delta}^{E_{1}}\right)$. Then the first order optimality conditions for $\left(P_{\varepsilon \delta}^{E_{1}}\right)$ are the following

$$
\left\{\begin{aligned}
&\left(u-u^{*}, p^{*}\right)_{L^{2}((0,1))} \geq 0 \quad \forall u \in U_{a d}, \\
&\left(v-v^{*}, y^{*}-q+\varepsilon p^{*}\right)_{L^{2}((0,1))} \leq 0 \quad \forall v \leq 0
\end{aligned}\right.
$$

where $p^{*}$ is the weak solution of the following adjoint equation

$$
\left\{\begin{aligned}
p^{* i v} & =y^{*}-q-\frac{1}{\varepsilon} v^{*}-\frac{1}{\delta}\left[y^{*}-q\right]^{-} \\
p^{*} & \in H_{0}^{2}((0,1))
\end{aligned}\right.
$$

In numerical tests we have used an equidistant partition of $[0,1]$, that is $0=a_{0}<a_{1}<$ $, \ldots,<a_{N}=1, a_{i}-a_{i-1}=h, i=1, \ldots, N$. The trapedzoidal rule have been applied for the numerical integration on each interval $\left[a_{i}, a_{i+1}\right], i=1, \ldots, N$. The discrete function spaces for $u_{h}, y_{h}$ and $v_{h}$ are given as follows

$$
\begin{aligned}
& U_{a d}^{h}=\left\{u_{h} \in L^{2}([0,1])\left|u_{h}\right|_{\left[a_{i-1}, a_{i}\right]} \in P_{0}, i=1, \ldots, N\right\} \cap U_{a d}, \\
& H^{h}=\left\{y_{h} \in C^{1}((0,1))\left|y_{h}\right|_{\left[a_{i-1}, a_{i}\right]} \in P_{3}, y_{h}(0)=y_{h}^{\prime}(0)=y_{h}(1)=y_{h}^{\prime}(1)=0\right\}, \\
& V^{h}=\left\{v_{h} \in C([0,1])\left|v_{h}\right|_{\left[a_{i-1}, a_{i}\right]} \in P_{1}, i=1, \ldots, N\right\} .
\end{aligned}
$$


For optimization we have used NAG-library subroutine E04UCF, which is based on the sequential quadratic programming method. The gradients for the optimization have been evaluated analytically by using the adjoint equation technique. In each test $\varepsilon=3 * 10^{-3}$, $\delta=10^{-12}, \alpha=-12, \beta=0, M=-3, q \equiv-0.001$ and the initial guess $u_{i}=M$ and $v_{i}=0$. The test runs have been performed in HP9000/720 computer with accuracy approximately 16 decimal digits.

The next figures represents the decreasing of the cost function $J_{\varepsilon \delta}$ versus iteration steps and the optimal values of $-u_{h}, v_{h}$ and $y_{h}$. The meaning of the numerical values in the figures are the following (integration means here the trapezoidal rule):

- OBJF final cost value, where the term $\frac{1}{2} \int_{0}^{1}\left(y_{h}-q_{h}\right)^{2} d x$ is multiplied by $10^{6}$

- PENEPS final value of the term $-\frac{1}{\varepsilon} \int_{0}^{1} v_{h}\left(y_{h}-q_{h}\right) d x$

- PENDEL final value of the term $\frac{1}{2 \delta} \int_{0}^{1}\left(\left[y_{h}-q_{h}\right]^{-}\right)^{2} d x$

- ROBJF value of the term $\frac{1}{2} \int_{0}^{1}\left(y_{h}-q_{h}\right)^{2} d x$ multiplied by $10^{6}$

- ITER number of iterations

The Figure 4.2. represents the solution with initial control $u_{h}$. The Figures 4.3. ,.., 4.7. represents the optimal solution with various discretization. In the Figures 4.8.1. ,.., 4.8.4 we see the development of the optimization with $N=160$ (optimal solution in Fig. 4.7.).
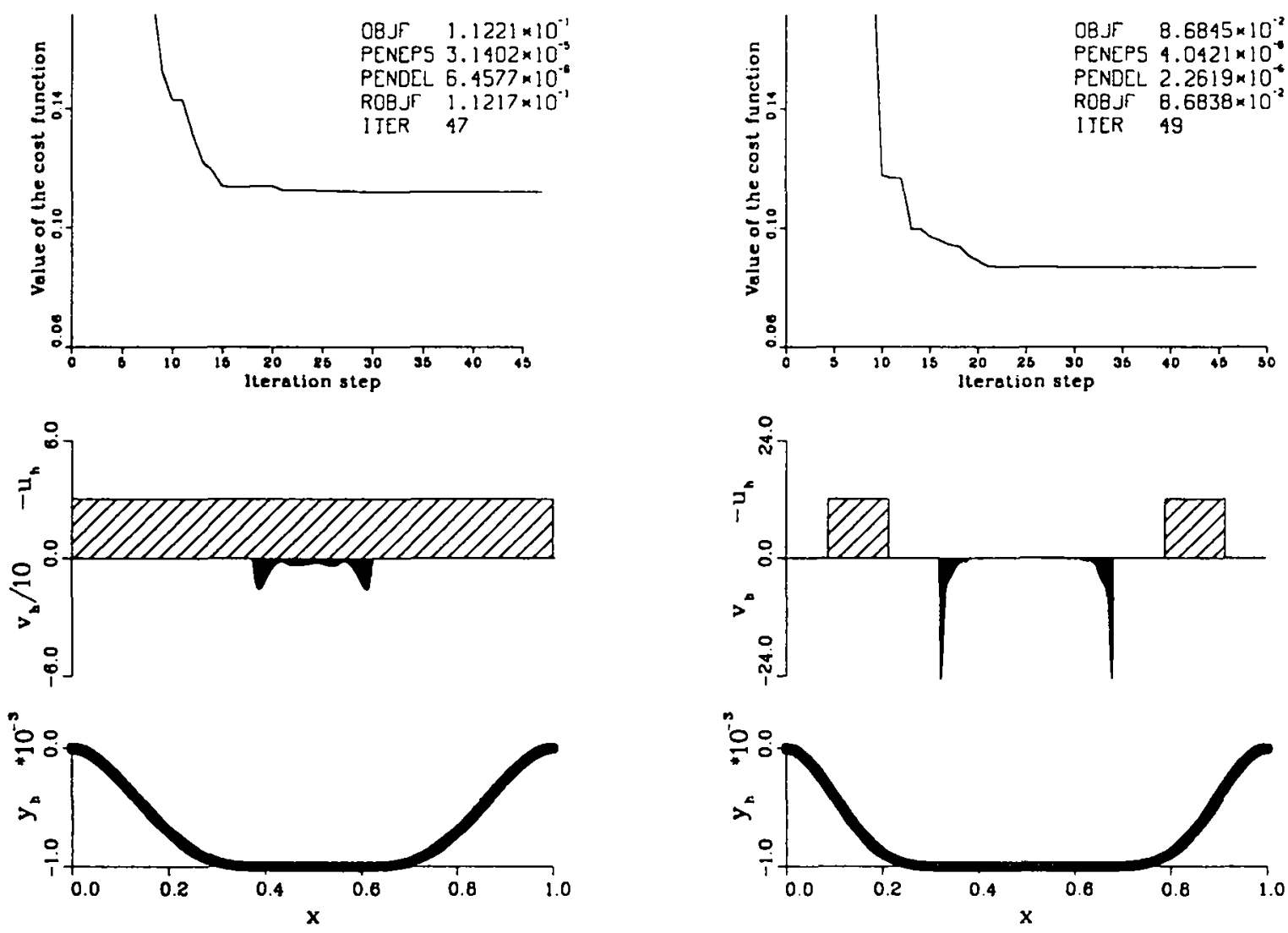

Figure 4.2. Solution with initial $u$ and $N=320$.

Figure 4.3. Optimal solution with $N=320$. 

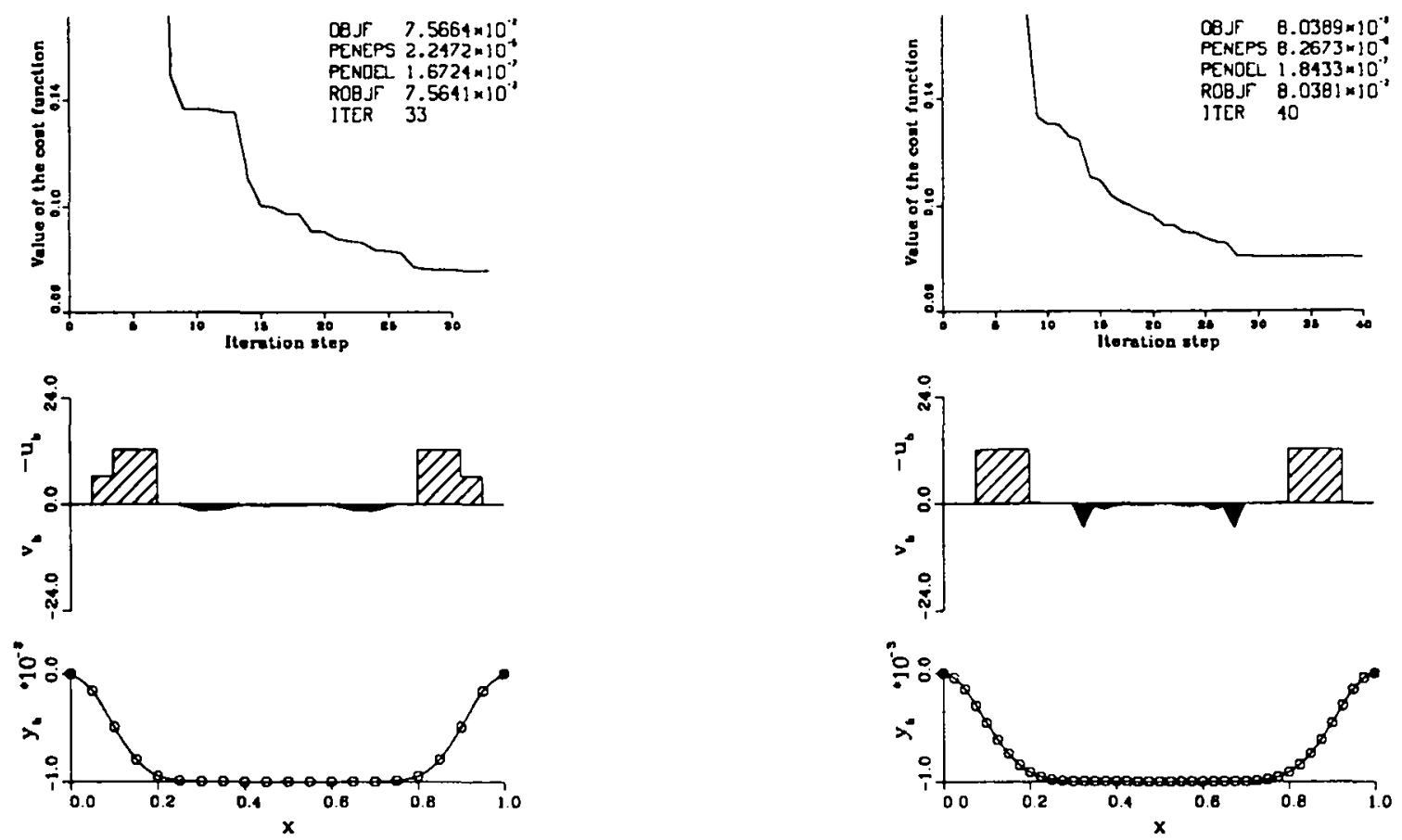

Figure 4.4. Optimal solution with $N=20$.
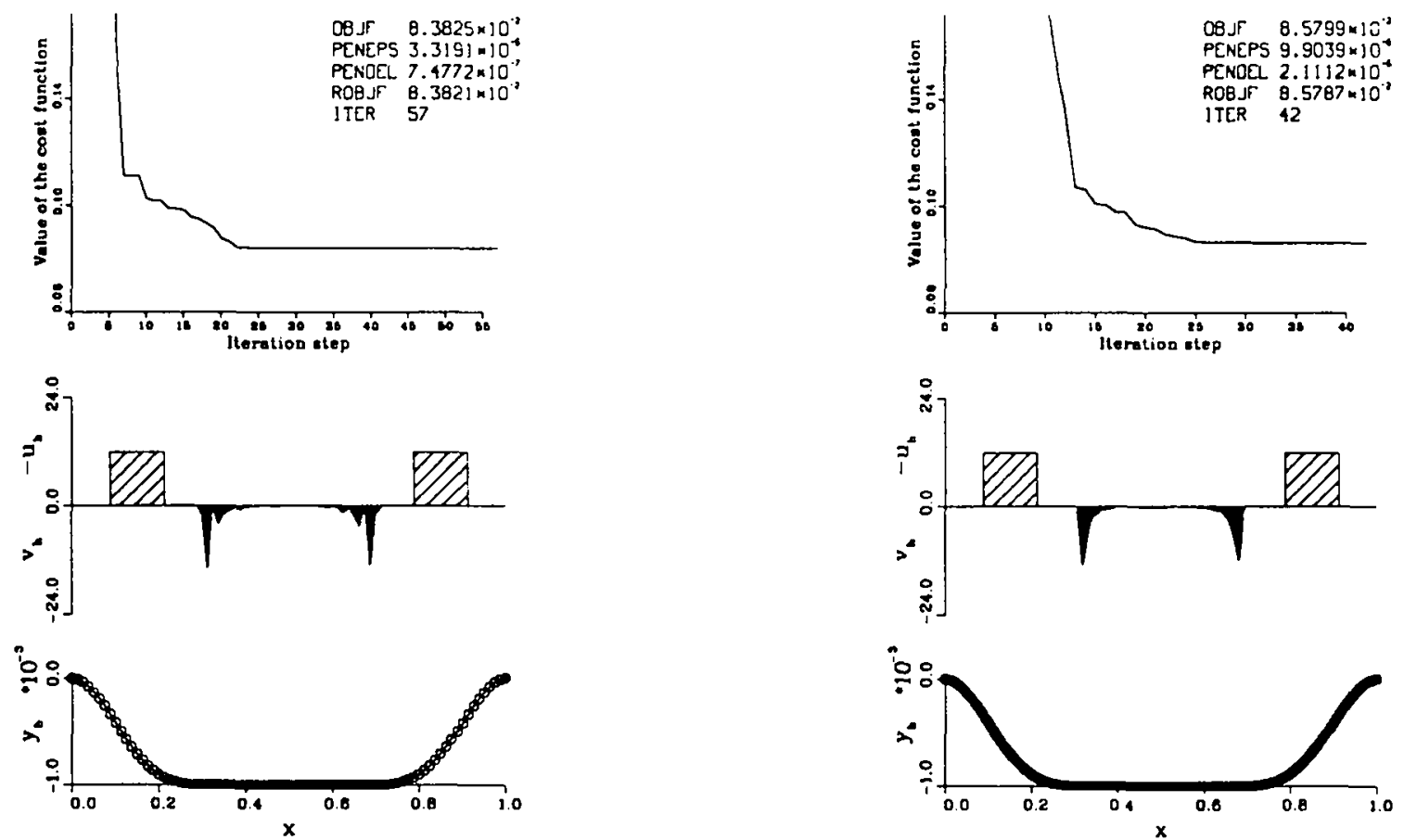

Figure 4.6. Optimal solution with $N=80$.

Figure 4.7. Optimal solution with $N=160$. 

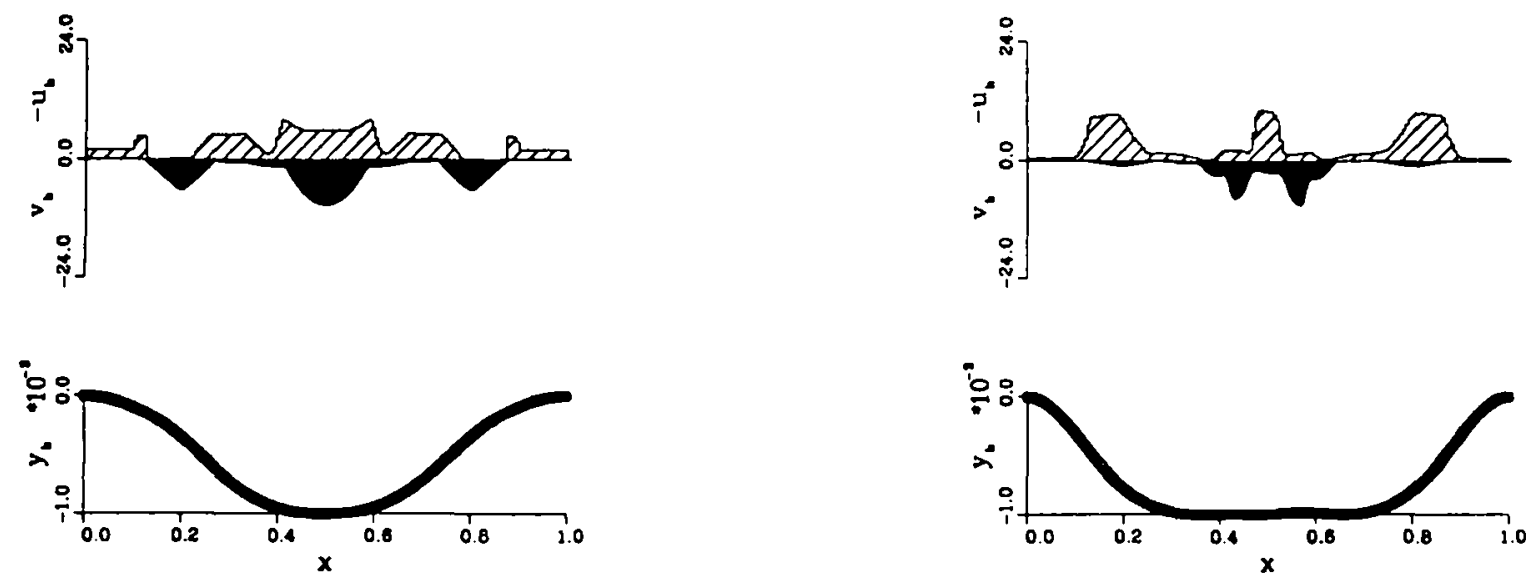

Figure 4.8.1. 10 steps $R O B J=0.182$.

Figure 4.8.2. 15 steps $R O B J=0.101$.
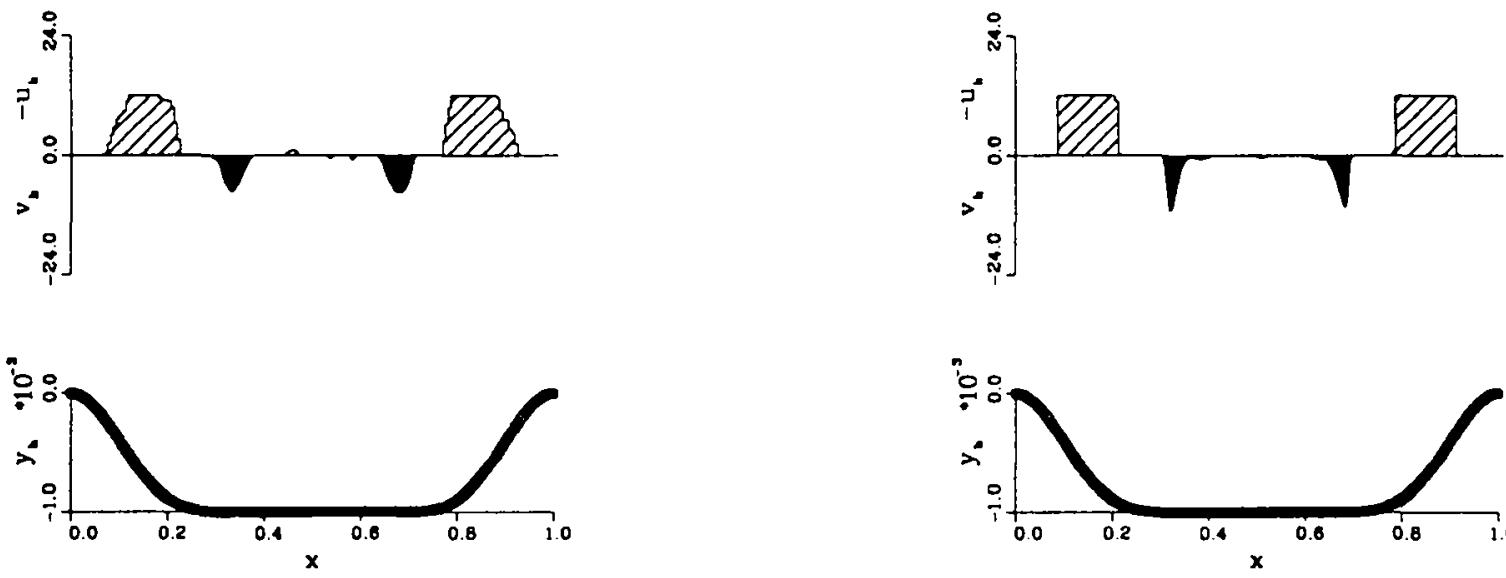

Figure 4.8.3. 25 steps $R O B J=0.0864$.

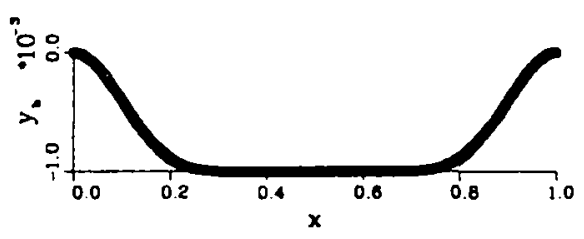

Figure 4.8.4. 35 steps $R O B J=0.0858$.

\section{EXAMPLE 2.}

In the second example we will replace the linear operator $A y=y^{i v}$ of the first example by the nonlinear operator $A(y)=\left(\left(y^{\prime}\right)^{3}+y^{\prime}\right)^{\prime}$. The example becomes then similar to the case where we have a loaded string insted of the beam (see Figure 4.9.).

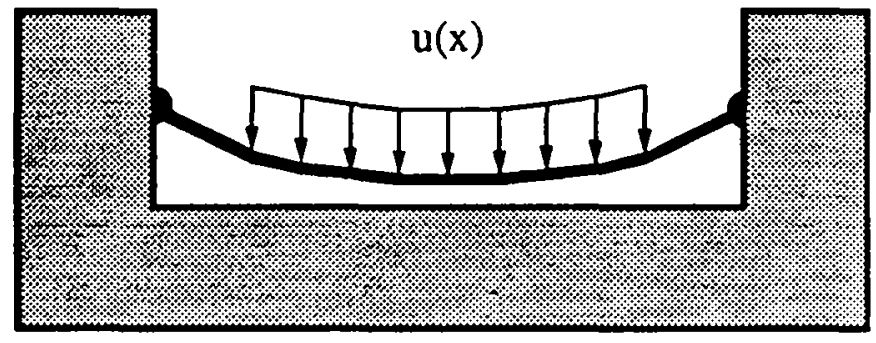

Figure 4.9. 
Denoting $\left(y^{\prime}\right)^{3}+y^{\prime}$ by $a(y)$ and $q(x) \equiv \psi=$ Const. we observe that the assumptions (3.15) and (3.16) are valid and we can write the corresponding problem for $\left(P_{\varepsilon \delta}^{E_{1}}\right)$ as follows

$$
\begin{aligned}
\underset{\substack{\operatorname{minimize} \\
v \in U_{a d}}}{v \leq 0}\left\{J_{e \delta}(y, v) \equiv\right. & \frac{1}{2} \int_{0}^{1}(y(x)-\psi)^{2} d x \\
& -\frac{1}{\varepsilon} \int_{0}^{1} v(x)(y(x)-\psi) d x \\
& \left.+\frac{1}{2 \delta} \int_{0}^{E_{2}}\left([y(x)-\psi]^{-}\right)^{2} d x\right\},
\end{aligned}
$$

where $y(x)$ is the variational solution for the equation

$$
\left\{\begin{aligned}
\left(\left(y^{\prime}\right)^{3}+y^{\prime}\right)^{\prime} & =u-v \\
y & \in W_{0}^{1,4}((0,1)) .
\end{aligned}\right.
$$

and

$$
U_{a d}=\left\{u \in L^{\infty}((0,1)) \mid \alpha \leq u(x) \leq \beta, \text { a.e. in }(0,1), \int_{0}^{1} u(x) d x=M\right\}
$$

with given positive constants $\alpha, \beta$ and $M$.

Let $\left(y^{*}, u^{*}, v^{*}\right)$ be optimal in $\left(P_{\varepsilon \delta}^{E_{2}}\right)$. Then according to Theorem 2 we have the following first order optimality conditions for $\left(P_{\varepsilon \delta}^{E_{2}}\right)$

$$
\left\{\begin{aligned}
\left(u-u^{*}, p^{*}\right)_{L^{2}((0,1))} & \geq 0 \quad \forall u \in U_{a d}, \\
\left(v-v^{*}, y^{*}-\psi+\varepsilon p^{*}\right)_{L^{2}((0,1))} \leq 0 & \forall v \leq 0,
\end{aligned}\right.
$$

where $p^{*}$ is the weak solution of the following adjoint equation

$$
\left\{\begin{aligned}
\left(\left(3\left(y^{* \prime}\right)^{2}+1\right) p^{* \prime}\right)^{\prime} & =y^{*}-\psi-\frac{1}{\varepsilon} v^{*}-\frac{1}{\delta}\left[y^{*}-\psi\right]^{-} \\
p^{*} & \in W_{0}^{1,4}((0,1)) .
\end{aligned}\right.
$$

In numerical tests the discrete function spaces for $u_{h}$ and $v_{h}$ are same as in Example 1 and the space for $y_{h}$ is given as follows

$$
W^{h}=\left\{y_{h} \in C([0,1])\left|y_{h}\right|_{\left[a_{i-1}, a_{i}\right]} \in P_{1}, y_{h}(0)=y_{h}(1)=0\right\} .
$$

Again the trapezoidal rule have been used for numerical integration. The discrete nonlinear state equation was numerically solved by using Newton -method. In each test $N=160$, $\varepsilon=10^{-4}, \delta=0.5 * 10^{-10}, \beta=0, M=-0.01, \psi=-0.001$ and the initial guess $u_{i}=M$ and $v_{i}=0$. The next figures represents the solutions with various $\alpha$ (lower bound of the control). 

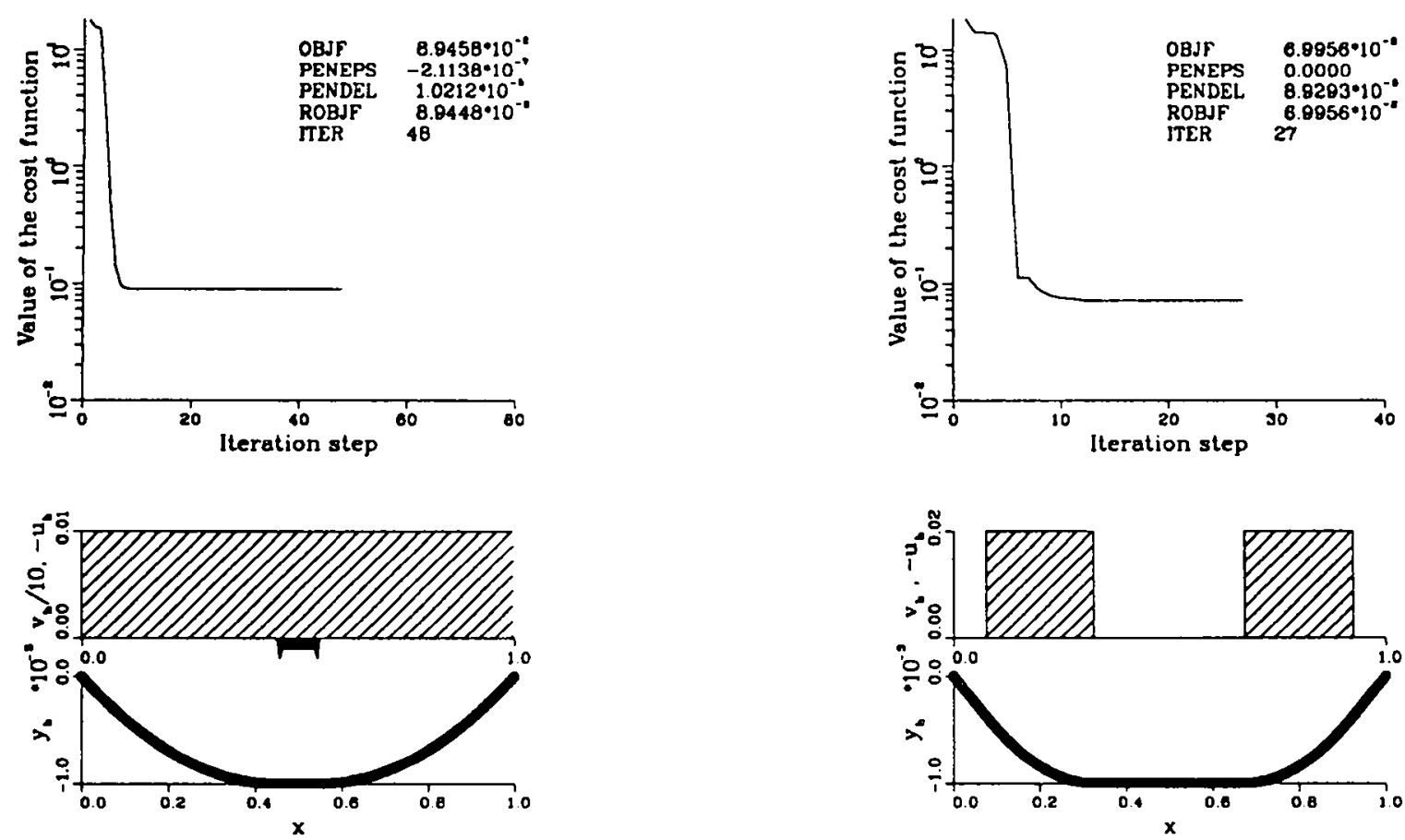

Figure 4.10. Solution with $\alpha=-0.01$.

Figure 4.11. Solution with $\alpha=-0.02$.
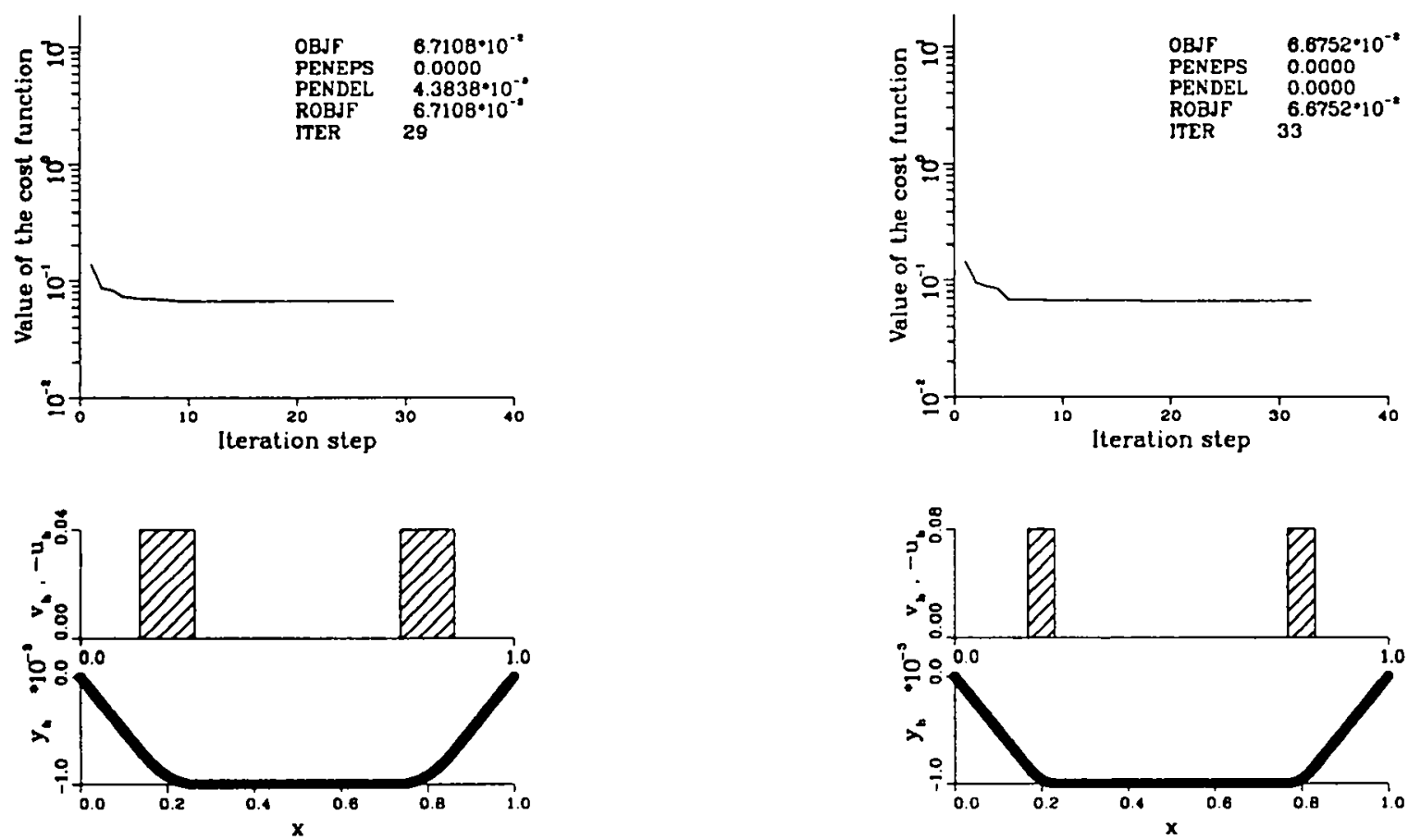

Figure 4.12. Solution with $\alpha=-0.04$.

Figure 4.13. Solution with $\alpha=-0.08$. 

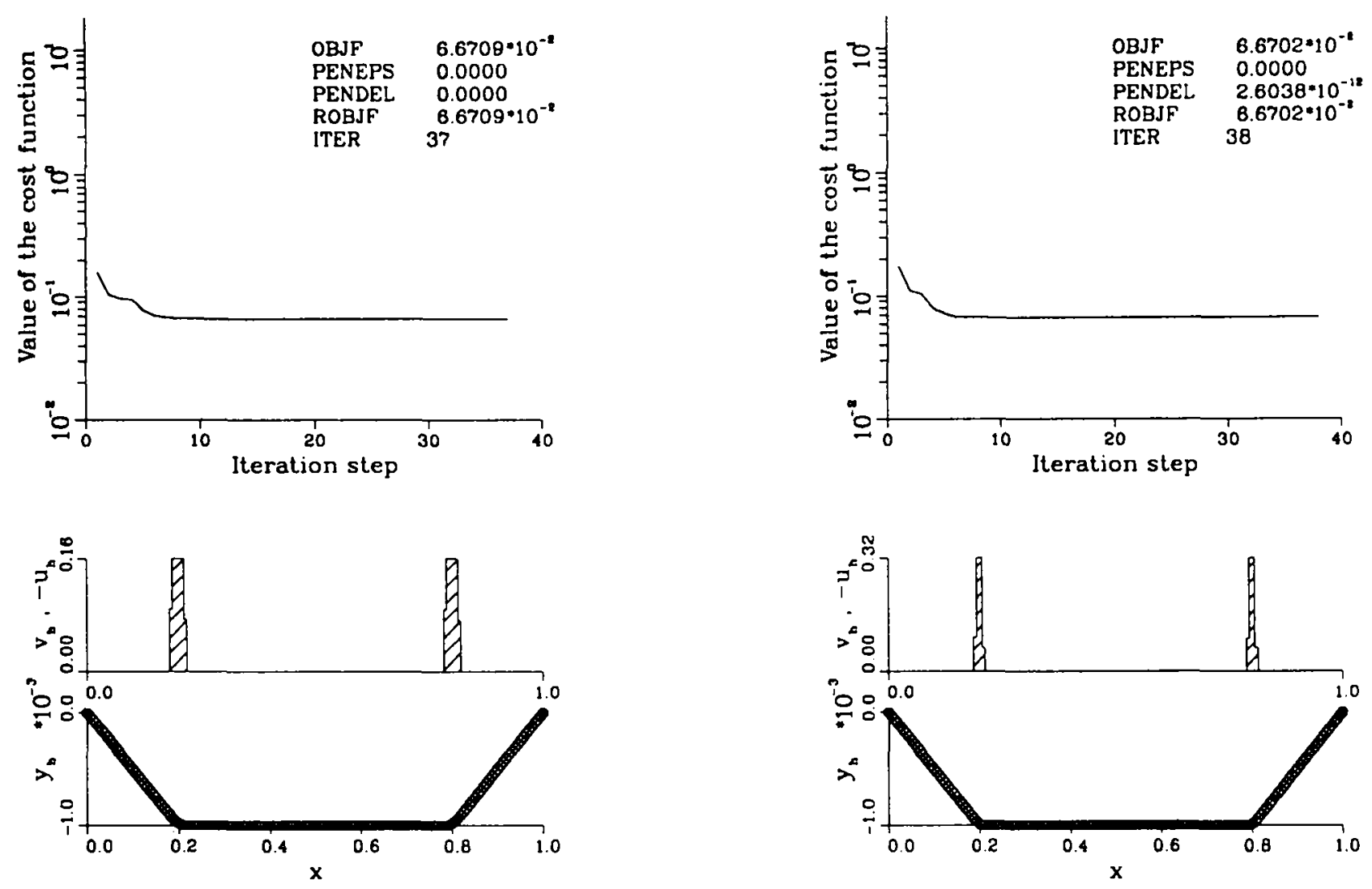

Figure 4.14. Solution with $\alpha=-0.16$.

Figure 4.15. Solution with $\alpha=-0.32$.

REMARK 1. Example 1 has been treated in [5] and [6]. In [5] the exterior penalty technique was applied to handle the state constraint and in [6] the exact penalty technique was applied.

\section{REFERENCES}

1. V. Barbu, "Optimal Control of Variational Inequalities," Research Notes in Mathematics 100, Pitman, London, Boston, Melbourne, 1984.

2. V. Barbu and T. Precupanu, "Convexity and Optimization in Banach Spaces," D. Reidel Publishing Company, Dordrecht, Boston, Lancester, 1986.

3. V. Barbu and D. Tiba, Optimal control of abstract variational inequalities, in "Control of Distributed Parameter Systems," Amouroux et El Jai Eds., Pergamom Press, Oxford, 1989.

4. H. Brezis, "Operateurs Maximaux Monotones et Semigroupes de Contractions Dans Les Espaces de Hilbert," Math. Studies 5, North-Holland, 1973.

5. J. Haslinger and P. Neittaanmäki, "Finite element approximation for optimal shape design. Theory and applications," John Wiley \& Sons, Chichester, 1988.

6. M. Mäkelä and P. Neittaanmäki, "Nonsmooth optimization. Theory and applications to optimal control," World Scientific Publisher, Singapore, 1992. 
ISSN 0249-6399 